\title{
Literature Review on Energy Minimization with Effective Data Partitioning for Fail Safe Cloud Storage and Computation
}

\author{
Zareena Sameer \\ Research Scholar \\ Department of Computer Science, \\ College of Engineering Perumon
}

\author{
Anoop S. \\ Assistant Professor \\ Department of Information Technology \\ College of Engineering Perumon
}

\begin{abstract}
Mobile Cloud Computing is an inheritance and emergence of cloud computing and mobile computing. The ever increasing usage of mobile devices and the need for users to handle applications that require high computation power has lead to the emergence of mobile cloud computing. The full potential of a mobile cloud can be realized only if its inherent hurdles such as resource scarcity, mobility and node failures can be overcome.Even then, in the case of dynamic networks, the challenges of reliability and energy efficiency remain largely unaddressed.These two challenges are addressed in a combined manner by using $\mathrm{k}$-out of $\mathrm{n}$ computing.In the proposed scheme the mobile devices retrieve or process data in the most energy efficient and fail safe method by using effective data partitioning in the cloud and $\mathrm{k}$-out of $\mathrm{n}$ servers. The $\mathrm{n}$ servers are chosen based on their topology and fault tolerance of the nodes. The k-out of $n$ computing ensures that a system of $n$ components operate correctly as long as k or more components work
\end{abstract}

\section{General Terms}

Mobile Computing, Cloud Computing, Mobile Cloud

\section{Keywords}

Energy-Efficient Computing, Fault-Tolerant Computing

\section{INTRODUCTION}

Over the past few years, the increasing data and growth of applications have lead to the emergence of cloud computing.Cloud computing may be described as a range of services provided by an internet based group of systems. These servers provide data storage,computation and processing to the clients.

Meanwhile, the popularity and use of smartphones have increased tremendously.Mobile computing provides both ubiquity and mobility.But the ever increasing demand of users and data along with the resource scarcity has brought the growth of mobile computing to a standstill.To overcome this gigantic barrier the mobile cloud computing emerged.Mobile cloud computing augments mobile computing with the advantages of cloud computing thus rendering

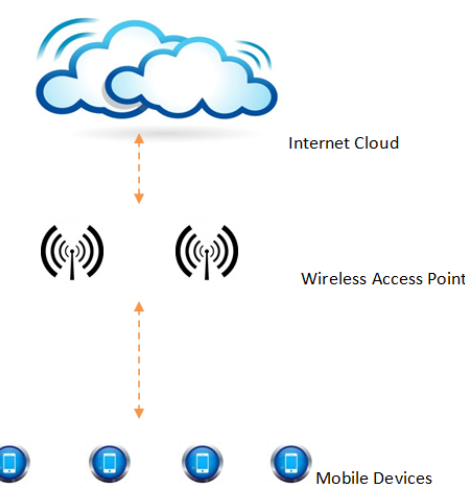

Fig. 1. Mobile Cloud Computing

its applications limitless. While mobile cloud computing brings up great promises there are a lot of challenges they have to overcome to seamlessly connect different technologies.

The paper organization is as follows. Section 2 presents a brief explanation of the core concepts of mobile cloud computing.Section 3 presents a comprehensive literature review about the current approaches in mobile cloud computing research. The criteria for defining the review is based on the key issues in mobile cloud computing.Section 4 gives a tabular representation of differnt node selection models.Finally, conclusion and future research are identified in Section 4.

\section{BACKGROUND}

(1) Cloud Computing

Mobile devices and smart phones are heavily used in today's world and their use is ever increasing with the emergence of mobile internet.The high demand for mobile applications has lead to the growth of the different applications for these devices. However these applications have to consider the resource scarceness of the devices such as processing power, available memory and energy consumption.. A technology recently emerged in the IT industry offers an opportunity to solve 


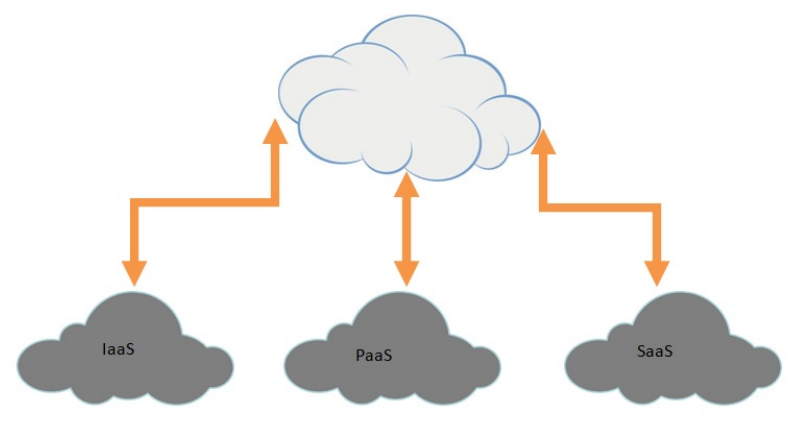

Fig. 2. Cloud Computing Services

these problems: Cloud computing (CC) gives its users the possibility to host and deliver services over the internet by dynamically providing computing resources. Thus cloud computing enables users to oversee the requirements of storage and computing power which is highly attractive to business owners. The Cloud computing services provided are:Infrastructure as a Service (IaaS), Platform as a Service(PaaS), and Software as a Service(SaaS). A recent Berkeley report [11] stated: "Cloud computing, the long-held dream of computing as a utility, has the potential to transform a large part of the IT industry, making software even more attractive as a service". Cloud computing allows IT companies to focus on innovation and services rather than setting up hardware and software infrastructures. The availability of cloud computing services in a mobile environment, called mobile cloud computing, can offer a solution to the resource scarcity of mobile devices.

\section{(2) Mobile Cloud Computing}

The popularity of personal mobile devices and mobile computing has increased tremendously over the years. The reason for this popularity is that mobile computing provides a tool to the user when and where it is needed irrespective of user mobility, thus enabling them to continue his/her work seamlessly. However with mobility also comes their issue of resource scarceness, finite energy and low connectivity. Recent research has addressed these problems through cloud computing, thus providing users with real time response. High level of responsiveness are not only demanded by real time mobile applications but also by application like location based social networking which process and make use of the phones sensor data. But the price we have to pay is in terms of energy. Due to this reason there is a limit for developing applications that require high computational costs for mobile devices. The current trends in mobile architecture and battery do not promise a solution to these problems. Hence we have to approach this problem in another way to realize the full potential of mobile computing as these problems are a barrier that is intrinsic to mobility.

The concept of offloading data and computation in cloud is used to address the inherent problems in mobile computing by using resource providers other than the mobile device itself to host the execution of mobile applications. Such an infrastructure where data storage and processing could happen outside the mobile device could be termed a mobile cloud. Thus a mobile cloud enables the computer intensive applications to be executed on low resource mobile devices.
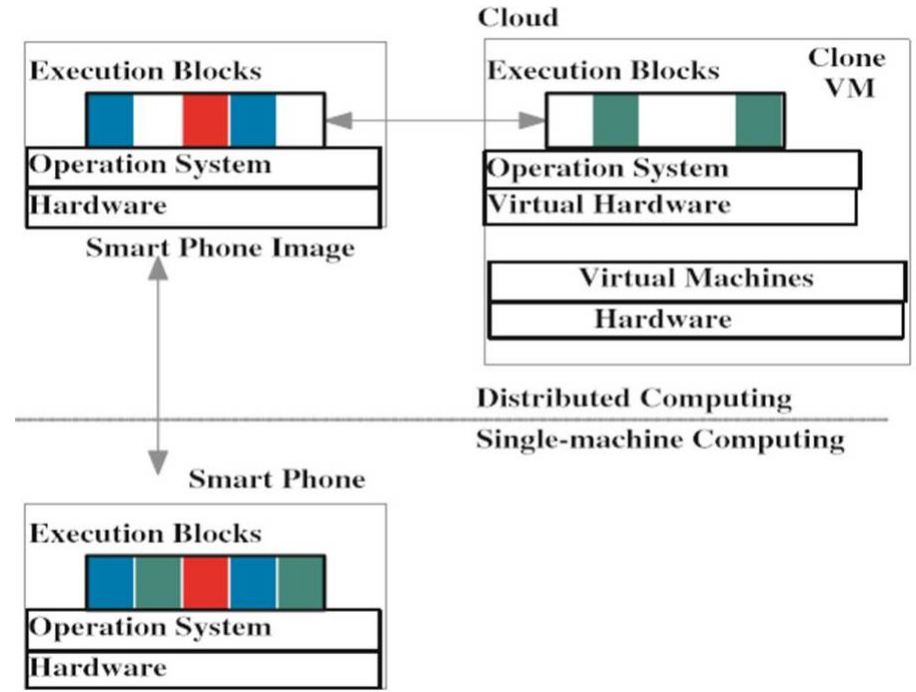

Fig. 3. Clone Cloud System Architecture [13]

\section{LITERATURE REVIEW}

There are many technical obstacles that mobile cloud computing has to overcome to achieve its true potential. The first and foremost obstacle is resource poverty. Although mobile hardware continues to evolve, it will always be resource poor compared to static hardware.

Virtual Machine migration was proposed to offload process and to liberate mobile devices from severe resource constraints. VM Migration implies transferring the memory image of a VM from a source server to destination server without stopping its execution, thus providing seamless connectivity. However VM migration could prove to be heavy for mobile devices and time consuming.

Instead of connecting to a distant cloud Satyanaranan et al [1] suggested cloudlets as a solution. A cloudlet is a cluster of multi-core computers that is connected to a larger cloud server through the internet. Thus the mobile deice in physical proximity to the cloudlet acts as a thin client while all the resource intensive computation takes place in the cloudlet. The mobile device and the cloudlet are connected by one hop high bandwidth wireless connection. The user can switch to the degraded service if he/she moves away from the cloudlet. The limitation with this technique is that it requires a complicated VM mechanism and a stable network connection. A combination of VM migration and code partitioning is used in MAUI [2]. the main objective of MAUI is to save energy. Here the partitioning is done at runtime and uses $3 \mathrm{G}$ or WiFi for connectivity. MAUI decides whether or not to offload methods at the time of execution.Mobile Assistance Using Infrastructure or MAUI performs as though the entire application is being executed on the mobile device while masking the complexity of remote execution from the user.The framework of MAUI is such that it adjusts with the mobility of the user as well as adapts to the changing network and user location. The most important aspect of MAUI is the dynamic partitioning of application at runtime, where the execution point for application partition is determined.Thus additional developmental efforts are required for creating applications suitable for MAUI. 
CloneCloud[3] also uses VM Migration for offloading workload to resourceful server. Here since device clones are used, the mobile applications are unmodified and the right portion of the code is offloaded into the device clones operating in the cloud.The central aspect of this method is to offload execution blocks of application from the device to cloud by using virtual machine migration. The clone in the clone cloud is a replica of the device running on a virtual machine. Thus unlike the mobile device the clone has more hardware, network,software and resources in a virtual machine which is more suitable for complex computations.Similar work can be seen in ThinkAir[10].Although there is an advantage of reduced power consumption since the CPU are used less frequently, the delay during handover and bandwidth delay are less acceptable.

All these papers addressed the problem of efficiency by providing different offloading methods to remote server.While considering local resources, the problems that are inherent in distributed computation also need to be considered, such as having data with boundaries so as to be easily partitioned, minimum time to recombine distributed data and also fault tolerance. Dimakis et al[9] proposed several erasure coding algorithms for dealing with the problem of fault tolerance.Another algorithm was proposed by Leong et al[4] that maximizes recover probability.Aguilera et al[5] proposed another scheme for efficiently adopting erasure code.

The abilities of a mobile cloud can be brought out if the issues it faces can be tackled together.But a solution to all the problems together is most probably a near impossible task.Current research has been able to tackle operational issues, energy issues,fault tolerance issues individually.

A combined solution to both energy efficiency and fault tolerance was put forth by Chien-An et al [8].The k-out of $n$ framework introduced a system that assigns data fragments to nodes in a way that other nodes can retrieve data reliably with minimum energy consumption. The framework also allows nodes to process the data such that the processing energy is also minimized.

\section{TABULAR REPRESENTATION OF DIFFERENT NODE SELECTION MODELS}

The advantages and disadvantages of the different methods by which nodes are selected are given in the following table.

Table 1. Tabular Representation of different node selection models

\begin{tabular}{|c|l|l|}
\hline Methods & Characteristics & Limitations \\
\hline Random & $\begin{array}{l}\text { selects storage or processor } \\
\text { nodes randomly }\end{array}$ & $\begin{array}{l}\text { The performance based on this } \\
\text { model is low.Low fault toler- } \\
\text { ance. }\end{array}$ \\
\hline Greedy & $\begin{array}{l}\text { selects nodes with the largest } \\
\text { number of neighbors as stor- } \\
\text { age/processor nodes }\end{array}$ & $\begin{array}{l}\text { Fault tolerance and efficiency } \\
\text { is lower than the random based } \\
\text { model }\end{array}$ \\
\hline $\begin{array}{c}\text { k-out- } \\
\text { of } \mathrm{n} \\
\text { rame- } \\
\text { work }\end{array}$ & $\begin{array}{l}\text { selects n nodes as stor- } \\
\text { age/processor nodes which } \\
\text { can operates correctly even if } \\
\text { only k of the components work } \\
\text { where } k<n\end{array}$ & Computation overhead is high \\
\hline
\end{tabular}

\section{CONCLUSION AND FUTURE WORK}

Empowering the mobile user is the capital aim of mobile cloud computing by providing them with seamless connectivity and rich functionality.The possible prospects of mobile cloud computing are numerous and will thus become the dominant model for mobile applications in the future.The $\mathrm{k}$-out of $\mathrm{n}$ framework used for node selection not only improves efficiency but also provides fault tolerance which is necessary in a dynamic network. This framework improves performance when compared to random node selection model and greedy node selection. Real time applications in mobile cloud require quick response times, thus attaining an optimal solution for data distribution and processing in mobile cloud becomes more necessary as we move into the future.

\section{REFERENCES}

[1] M. Satyanarayanan, P. Bahl, R. Caceres, and N. Davies, "The case for VM-based cloudlets in mobile computing," Pervasive Computing, IEEE, vol. 8, pp. 14-23, 2009.

[2] E. Cuervo, A. Balasubramanian, D.k Cho, A. Wolman,S. Saroiu, R. Chandra, and P. Bahl, "MAUI: making smartphones last longer with code offload," in Proc. of MobiSys, 2010.

[3] ]B.-G. Chun, S. Ihm, P. Maniatis, M. Naik, and A. Patti, "CloneCloud: elastic execution between mobile device and cloud," in Proc. of EuroSys, 2011.

[4] D. Leong, A. G. Dimakis, and T. Ho, "Distributed storage allocation for high reliability," in Proc. of ICC, 2010.

[5] M. Aguilera, R. Janakiraman, and L. Xu, "Using erasure codes efficiently for storage in a distributed system," in Proc. of DSN, 2005

[6] C. Chen, M. Won, R. Stoleru, and G. Xie, "Resource allocation for energy efficient k-out-of-n system in mobile ad hoc networks," in Proc. of ICCCN, 2013.

[7] C. A. Chen, M. Won, R. Stoleru, and G. Xie, "Energy efficient fault-tolerant data storage and processing in dynamic network," in Proc. of MobiHoc, 2013.

[8] C. A. Chen, M. Won, R. Stoleru, and G. Xie, 'Energy efficient fault-tolerant data storage and processing in mobile cloud," in IEEE Transactions on Cloud Computing, 2014.

[9] A. G. Dimakis, K. Ramchandran, Y. Wu, and C. Su, "A survey on network codes for distributed storage," Proc. of the IEEE, vol. 99, no. 3, pp. 476-489, 2010.

[10] S. Kosta, A. Aucinas, P. Hui, R. Mortier, and X. Zhang, "ThinkAir: Dynamic resource allocation and parallel execution in the cloud for mobile code offloading," in Proc. of INFOCOM, 2012.

[11] M. Armbrust, A. Fox, R. Griffith, A.D. Joseph, R. Katz, A Konwinski, G. Lee, D. Patterson, A. Rabkin, I. Stoica, M Zaharia, A view of cloud computing, CommunicationsoftheACM53(4)(2009)50?58.

[12] J.Kaplan,W.Forrest,N.Kindler,'Revolutionizing Data Center Energy Efficiency", McKinsey and Company,Tech.Rep.

[13] Han Qi,Abdullah Gani,'Research on Mobile Cloud Computing: Review, Trend and Perspectives",IEEE 2012 Second International Conference on Digital Information and Communication Technology and it's Applications (DICTAP) 